\title{
Low lying oscillations of deformed nuclei
}

\author{
Ani Aprahamian ${ }^{1,}{ }^{*}$ and Shelly R. Lesher ${ }^{2}$ \\ ${ }^{1}$ Physics Department, University of Notre Dame, Notre Dame, IN 46556, USA \\ ${ }^{2}$ Physics Department, University of Wisconsin-La Crosse, La Crosse, WI, 54601, USA
}

\begin{abstract}
Low-lying oscillations of the intrinsic deformed shape of a nucleus remain an open challenge in nuclear structure. The question or challenge revolves around the viability of single or multiple quanta of vibrational excitations superimposed on the equilibrium, deformed shape of a nucleus. The $\mathrm{K}=2$ or " $\gamma$ " vibrations are fairly widespread and nominally conform to expectations whereas the existence of the $\mathrm{K}=0$ or " $\beta$ " vibrational excitation is yet to be distinguished from other possible origins including the coexistence of other potential minima.
\end{abstract}

\section{Introduction}

The 1975 Nobel prize in Physics was awarded to Bohr, Mottelson, and Rainwater with the citation that read "for the discovery of the connection between collective motion and particle motion in atomic nuclei and the development of the theory of the structure of the atomic nucleus based on this connection". They were recognized for a geometric description of the nucleus from spherical, to deformed, and triaxial shapes with superimposed oscillations or vibrations of those shapes. There have been many advances since in describing nuclei in terms of sophisticated approaches and algebraic models but the question remains about the nature of the low lying oscillations in deformed nuclei. The lowest shape affecting oscillations are expected to be quadrupole in nature resulting in two types of vibrations, a " $\beta$ " and a " $\gamma$ " type of vibrations where one type of vibration is along the symmetry axis and the other is against it with a projection of $\mathrm{K}=2^{+}$on the symmetry axis [1]. The " $\gamma$ " type of vibration seems to be well characterized as the first excited $\mathrm{K}=2^{+}$ bands in deformed nuclei with typical B(E2) values of several Weisskopf units (W.u.) connecting states to the ground state (g.s.) band. Today, however, some forty years later, the nature of the " $\beta$ " or the $\mathrm{K}=0^{+}$excitation is still unsettled. The topic has been discussed, questioned, challenged and redefined in numerous publications. A part of the challenge lies in the paucity of data and also to a significant extent on the interpretation or characterization of what a " $\beta$ " vibration is expected to be. Fig. 1 [2] shows the experimental situation in the rare-earth region of the chart of nuclides. The situation is accepted and fairly predictable for the first excited $\mathrm{K}=2^{+}$bands but not so for the first excited $\mathrm{K}^{\pi}=0^{+}$band. Perhaps the most resounding criticism of labeling the first excited $\mathrm{K}=0^{+}$excitations as a " $\beta$ "

* Corresponding author : aapraham@nd.edu 
vibration built on the intrinsic ground state deformation, come from the coexistence approaches claiming that the first excited $\mathrm{K}=0^{+}$band is in fact the minimum for a competing shape and therefore coexisting minimum in the potential. Other approaches include calculations using a five dimensional collective Hamiltonian for the quadrupole vibrational and rotational degrees of freedom where instead of solving a potential in $\beta$ and $\gamma$, potential energy surfaces are calculated using Relativistic Mean Field theory [3-6]. In this approach, the first excited $\mathrm{K}^{\pi}=0^{+}$excitation often ends up being a mixture of many coexisting shapes. There is also the constrained-Hartree-Fock-Bogoliubov (CHFB) theory together with a mapping to the five dimensional collective Hamiltonian, using the Cogny D1S interaction in the nuclear Hamiltonian [7,8]. The aim ofthis approach is the development of a theory of nuclear structure that is universal and includes spectroscopic calculations for nearly two thousand nuclei. They find that many of the properties are strongly dependent on the intrinsic deformation. On average, the predicted energies of the $\gamma$ band or excited $\mathrm{K}=2^{+}$band are $12 \%$ higher than experiment whereas the predicted energies for the first excited $0^{+}$state are systematically $50 \%$ higher [7]. The character of the first excited $0_{2}^{+}$is interpreted either as a $\beta$-vibrational excitation or as shape coexistence depending on the relative quadrupole transition strengths. The result is a prediction of $0^{+}$ bands with $\beta$ vibrational character for many well-deformed nuclei with $4^{+} / 2^{+}$ratios approaching that of the axial rotor at $10 / 3$ although they find coexistence to be more prevalent.

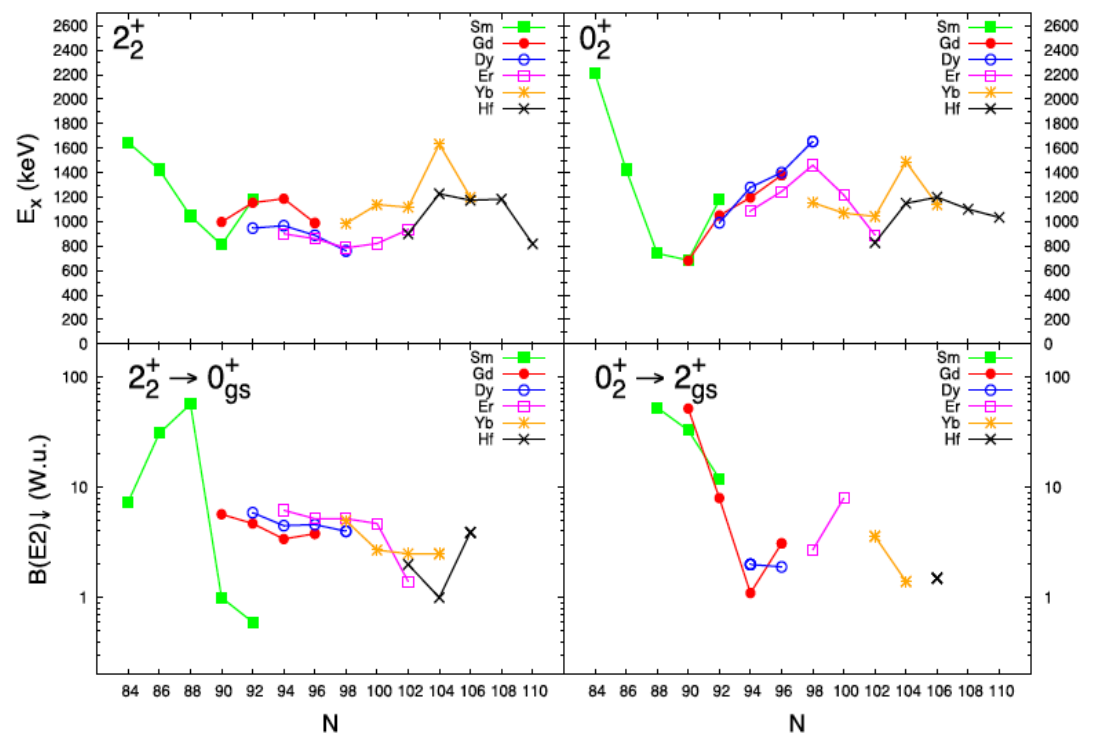

Figure 1: Systematics of the first excited $\mathrm{K}^{\pi}=2^{+}$“ $\gamma$ " and $\mathrm{K}^{*}=0^{+}$bands in several rare earth nuclear isotopes as a function of the neutron number $N$ along with their corresponding $B(E 2)$ values connecting the shown excited bands to members of the ground state band [2].

Our goal in this work is to discern the nature of the low-lying excited $0^{+}$states in deformed nuclei. The Gd nuclei lie in the well-deformed rare earth region of the chart of nuclides with $4^{+} / 2^{+}$energy ratios that vary between 3.0 and 3.33. In an earlier study [9], we had uncovered the existence of thirteen $0^{+}$states in ${ }^{158} \mathrm{Gd}$ with experiments at the Munich Q3D spectrometer. Here, we report on lifetime measurements of states in the ${ }^{156} \mathrm{Gd}$ nucleus. The 
$4^{+} / 2^{+}$energy ratio is 3.27 and there are three low-lying $0^{+}$bands. We report on measurements of lifetimes and the extracted quadrupole transition strengths for 8 members of the three excited $\mathrm{K}=0^{+}$bands using the Gamma-Ray-Induced Doppler (GRID) broadening technique [10] following neutron capture at the Institut Laue Langevin in Grenoble, France. The results point to an excited $\mathrm{K} \mathrm{O}^{+}{ }_{2}$ collective excitation built on the g.s. band in comparison to a nearby and much weaker $\mathrm{K}=0_{3}^{+}$excitation. An additional surprise of a third excited $\mathrm{K}=0^{+}{ }_{4}$ band emerged as a collective excitation built on the $\mathrm{K}=2^{+}$ band. We evaluate the various excitation bands in terms of quadrupole transition probabilities as well as dynamic moments of inertia.

\section{Experiments}

The experiment to measure lifetimes was performed at the High Flux Reactor at the Institute Laue-Langevin in Grenoble, France. GRID allows the measurement of the broadening of a given transition affected by the recoil of a previously emitted $\gamma$-ray. The technique uses nearly perfect crystals of Si and Ge to measure line shapes of the transitions of interest. The recoil velocities are very small $\left(10^{-4}\right.$ to $\left.10^{-6} \mathrm{c}\right)$ and the measured broadenings are in the order of a few keV. Typically, there are very few known lifetimes of the $0^{+}$band heads in nuclei and in this case, we were able to measure all three. Additionally we re-measured some previously known lifetimes in the $\mathrm{K}=2^{+}$band.

\section{Results}

The resulting transition probabilities for the ${ }^{156} \mathrm{Gd}$ from the lifetime measurements are shown in Fig. 2 along with the systematics of the neighboring isotopes of ${ }^{154,158,160} \mathrm{Gd}$. There are numerous $0^{+}$states identified in ${ }^{154} \mathrm{Gd}$ but lifetimes are not yet available. We report on the results for ${ }^{156} \mathrm{Gd}$ here. Lifetimes of levels in ${ }^{158,160} \mathrm{Gd}$ were measured using $(n, n ' \gamma)$ method at the University of Kentucky.

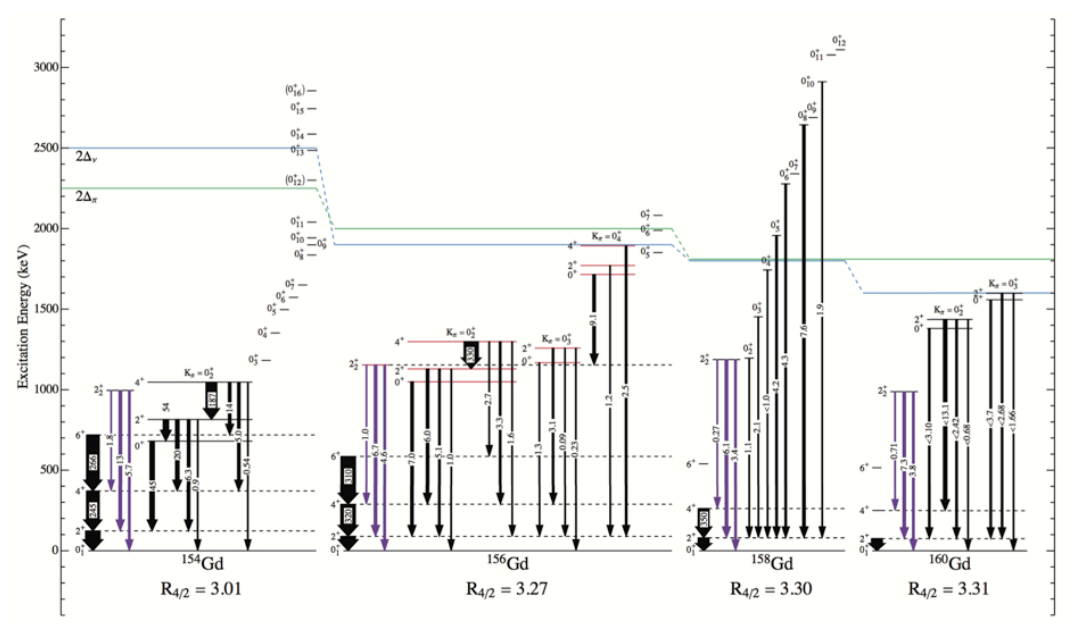

Figure 2: Partial level schemes are shown for the isotopes of ${ }^{154,156,158,160} \mathrm{Gd}$. The $\mathrm{B}(\mathrm{E} 2)$ values from the lowest $\mathrm{K}=2^{+}$bands are included as well as the $\mathrm{K}=0^{+}$bands where lifetimes are available. The widths of the arrows are proportional to the $B(E 2)$ strengths and given in W.u. 
Below the isotope label are the energy ratios of the first $4^{+} / 2^{+}$values. The horizontal lines represent the two proton and two neutron pairing gaps.

\section{Discussion}

The $\mathrm{B}(\mathrm{E} 2)$ values for transitions depopulating the first excited $\mathrm{K}=0^{+}$band in ${ }^{156} \mathrm{Gd}$ are of the same order as those depopulating the first excited $\mathrm{K}=2^{+}$band. Indicating that the first excited $\mathrm{K}=0^{+}{ }_{2}$ band may in fact be a " $\beta$ " vibrational excitation built on the ground state in this nucleus. We have further evidence that the second $\mathrm{K}=0^{+}$band is much more weakly connected to the ground state levels while the third excited $\mathrm{K}=0^{+}{ }_{4}$ band is strongly connected to the $\mathrm{K}=2^{+}$band. This latter band behaves as the two-phonon excitation built on the single phonon " $\gamma$ " vibrational excitation. In ${ }^{156} \mathrm{Gd}$, there had been an earlier measurement of the lifetime of the $\mathrm{K}^{\pi}=4^{+}$band head by coulomb excitation. $\mathrm{B}(\mathrm{E} 2)$ values depopulating this state to the $\mathrm{K}^{\pi}=2^{+}$band are strong however decay of the $\mathrm{K}^{\pi} 4^{+}$band to the g.s. band is $\mathrm{K}$ forbidden having no other open channels for decay. Also, the energy ratio of the $\mathrm{K}^{\pi}=4^{+} / \mathrm{K}=2^{+}$bands is strongly anharmonic with a ratio of 1.31 instead of the expected harmonic value of 2 . The $\mathrm{K}^{\pi}=0^{+}{ }_{4}$ band starting at 1715.181 however is strongly connected to the $\mathrm{K}=2+$ band as well and shows an energy ratio of approximately 1.5 . This is also strongly anharmonic but all evidence points to the band at $1715.181 \mathrm{keV}$ to be the twophonon $\gamma \gamma$ vibrational excitation built on the $\mathrm{K}^{\pi}=2^{+}$band. Earlier works $[11,12]$ had pointed to the existence of identical dynamic moments of inertia for bands of similar character including the case for the ${ }^{156} \mathrm{Gd}, \mathrm{K}^{\pi}=2^{+}$and $\mathrm{K}^{\pi}=4^{+}$bands. Here we include the new $\mathrm{K}^{\pi}=0^{+}{ }_{4}$ band shown to be the two-phonon " $\gamma \gamma$ " vibrational excitation built on the " $\gamma$ " band by the collective $\mathrm{B}(\mathrm{E} 2)$ values, in addition to the $\mathrm{K}^{\pi}=4^{+}$band in Fig. 3. The dynamic moments of inertia for single $\mathrm{K}^{\pi}=2^{+}$and two phonon vibrational bands $\left(\mathrm{K}^{\pi}=0^{+}, \mathrm{K}^{\pi}=4^{+}\right)$. The somewhat puzzling point is that the $\mathrm{K}^{\pi}=0^{+}$band does not have the same dynamic moment of inertia as the $\mathrm{K}^{r}=4^{+}$band in spite of the collective $\mathrm{B}(\mathrm{E} 2)$ values connecting them both to the $\mathrm{K}^{r}=2^{+}$ band. The 5DCH calculations within RMF approach show identical moments of inertia for the g.s. band and the gamma band but no information regarding the two-phonon excitation bands.

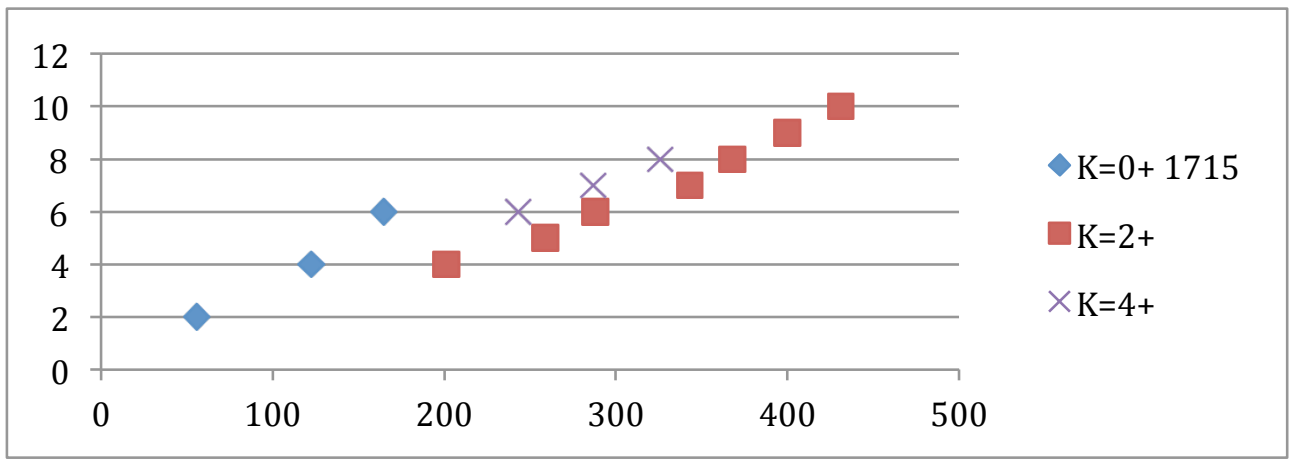

Figure 3: A plot of the initial spins against the level energy differences for the individual levels within a given band. Only $\Delta \mathrm{J}=2$ transitions are considered here. The dynamic moment of inertia for each band in ${ }^{156} \mathrm{Gd}$ is the slope of the band. This figures shows that the $\mathrm{K}^{\pi}=2^{+}$and $\mathrm{K}^{\pi}$ 
$=4^{+}$bands have identical slopes but the $\mathrm{K}^{\pi}=0^{+}$band at $1715 \mathrm{keV}$ has a slightly different slope if the uncertain $\left(6^{+}\right)$state is also included in spite of showing strong $B(E 2)$ transitions connecting the band to the $\mathrm{K}=2^{+}$band.

Following on the dynamic moments of inertia for the three $\mathrm{K}=0+$ bands, Fig. 4 below shows the relation of the three excited $\mathrm{K}=0^{+}$bands. The $\mathrm{K}=0^{+}$band at $1049.5 \mathrm{keV}$ connecting with collective transitions to the ground state band has the same slope as the $\mathrm{K}=0^{+}$band starting at $1715 \mathrm{keV}$ which is connecting to the $\mathrm{K}=2^{+}$band with collective transitions. Furthermore, the g.s. band dynamic moment of inertia is almost exactly identical to the $\mathrm{K}=0^{+}$at band starting at $1168 \mathrm{keV}$ however the quadrupole transitions connecting them is considerably weaker.

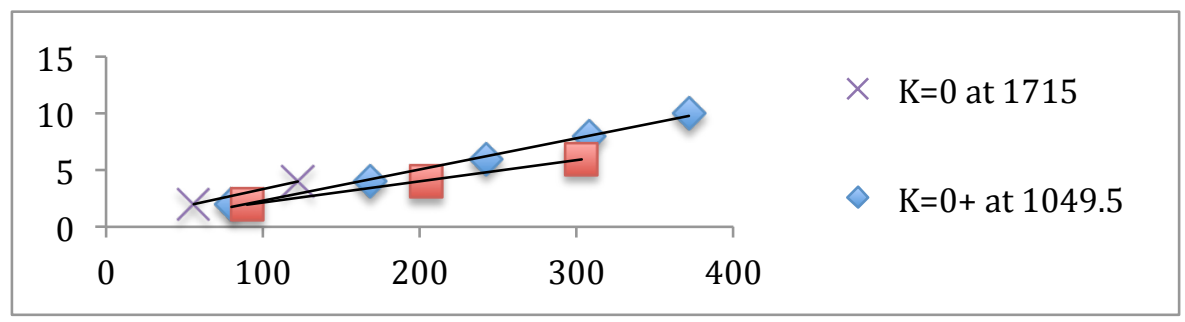

Figure 4: A plot of the initial spins ( $J$ ) plotted against the $\Delta J=2$ of the energy levels within a given band for the three excited $\mathrm{K}^{\pi}=0^{+}$bands in ${ }^{156} \mathrm{Gd}$. All indications here are that the $\mathrm{K}^{\pi}=0^{+}$ at $1168 \mathrm{keV}$ is a band of a very different nature than the other two $\mathrm{K}^{\pi}=0^{+}$bands at 1049.5 and $1715 \mathrm{keV}$.

\section{Conclusions}

We have reported on the lifetime measurements of three $\mathrm{K}^{\pi}=0^{+}$bands in the well-deformed ${ }^{156} \mathrm{Gd}$ nucleus. $\mathrm{B}(\mathrm{E} 2)$ transitions indicate that the first excited $\mathrm{K}^{\pi}=0^{+}$band is more strongly connected to the g.s. band than the nearby but higher $\mathrm{K}^{\pi}=0^{+}$band starting at $1168 \mathrm{keV}$. The $\mathrm{K}^{\pi}=0^{+}$band starting at $1715 \mathrm{keV}$ shows strong $\mathrm{B}(\mathrm{E} 2)$ transitions to the first excited $\mathrm{K}^{\pi}=2^{+}$ band. The dynamic moments of inertia for the bands in consideration show somewhat puzzling results.

This work was funded by the NSF-USA under contracts PHY-1419765 and PHY-1713857. References:

1. A. Bohr and B.R. Mottelson, Nuclear Structure Vol. II ,(1975)

2. A. Aprahamian, S.R. Lesher, and A. Stratman, Bulg. J. Phys. 44, 372 (2017)

3. A. Aprahamian et al., Phys. Rev. C 95, 024329 (2017).

4. T. Nikšić et al.,Phys. Rev. C 79, 034303 (2009)

5. Z. P. Li et al., Phys. Rev. C 79, 054301 (2009)

6. J.P. Delaroche et al, ,Phys. Rev. C 81, 014303 (2010)

7. J. Decharge and D. Gogny, Phys. Rev. C21, 1568 (1980)

8. G. F Bertsch et al, , Phys. Rev. Lett. 99, 032502 (2007)

9. S.R. Lesher et al, Phys. Rev. C 66, 051305 (R) (2002)

10. H.G. Boerner et al., Phys. Lett. B 215, 45 (1988)

11. X. Wu, A. Aprahamian, J. Castro-Ceron, Phys. Lett. B 316, 235 (1993)

12. A. Aprahamian et al., Phys. Rev. C 65, 031301 (R) (2002) 